\title{
Studien über die Eiweisskörper des Knochenmarks.
}

\author{
II. Mitteilung: Die Veränderungen der Eiweissfraktionen des \\ Knochenmarks bei experimenteller Nieren- und \\ Leberschädigung. \\ Von \\ Yutaka Nihei \\ (二㼟 泰) \\ (Aus der Medizinischen Klinik von Prof. Dr. T. Kato, \\ Tohoku Universität zu Sendai.) \\ (Received for publication, May 2, 1943)
}

Dass die Niere nicht nur Endprodukte des Stoffwechsels ausscheidet, sondern auch an der Regulation des Bluteiweissbildes beteiligt ist, geht aus Untersuchungen von $\mathrm{Häbler,} \mathrm{Günther,} \mathrm{Scheiner,} \mathrm{Wagner} \mathrm{u.}$ Wörne ${ }^{1)}$ und den Arbeiten hiesiger Klinik von K. Kimura u. Nakazawa, ${ }^{2}$ Sanada, ${ }^{3)}$, Shida ${ }^{4}$ u. a. einleuchtend hervor. Es ist eine bekannte Tatsache, dass bei Niereninsuffizienz die Funktion des Knochenmarks gestört wird und öfters Anämie zustande kommt. Wenn die Niere so an der Regulation des Bluteiweisses teilnimmt und ihre Insuffizienz die Funktion des Knochenmarks stört, muss es von grösstem Interesse sein, das Eiweiss im Knochenmark bei Nierenschädigung zu prüfen.

Es ist von vielen Autoren anerkannt, dass die Leber bei verschiedenen Stoffwechseln, insbesondere bei Eiweisstoffwechsel eine beherrschende Stellung einnimmt. Yasud $\mathrm{a}^{5}$ hat an hiesiger Klinik den Eiweissgehalt und den k.o.D. in dem der Leber zufliessenden und von ihr abfliessenden Blut bestimmt und festgestellt, dass die Leber bei Funktionsstörung in grossen Mengen grossmolekulare Eiweissteilchen ins Blut abgibt. Nolf, ${ }^{6)}$ Whipple u. Hurwitz, ${ }^{7}$ Tanaka, ${ }^{8}$ Mann u. Magath ${ }^{9}$ u.a. meinten, die Leber sei ein Bildungsorgan des Bluteiweisses. Da die Leber ein Organ ist, welches sich an der Regulation des Bluteiweisses stark beteiligt, und bei ihrer Schädigung Anomalien des Eiweisstoffwechsels auftreten, kann man sich leicht'vorstellen, dass das Knochenmark, welches in enger Beziehung zum Eiweisstoff wechsel steht, dadurch ebenfalls irgendwie beeinflusst wird.

Daher sind vorliegende Versuche vorgenommen, um den Eiweissspiegel des Knochenmarks bei Nieren- bzw. Leberfunktionsstörungen zu untersuchen. Da die Methode der Bestimmungen dieselbe war, wie bei den Untersuchungen der I. Mitteilung, wird sie hier nicht beschrieben. 


\section{Bestimmungen bei Nierenschädigung.}

Damit die direkte Funktionsinsuffizienz hauptsächlich bei der.Niere allein zustande kommen konnte, wurden keine Einspritzungen giftiger Substanzen oder dergleichen, sondern die beiderseitigen Ureterenligatur ausgeführt. Nach 48 Stunden, wenn die urämischen Symptome dadurch manifest auftraten, wurden die Versuchstiere untersucht. Die Ergebnisse sind in Tab. I angegeben.

TA B. I.

Eiweissverteilung im Blutplasma u. Knochenmark bei Ureterenunterbindung.

\begin{tabular}{|c|c|c|c|c|c|c|c|c|c|c|c|c|c|c|}
\hline \multicolumn{9}{|c|}{ Blutplasma } & \multicolumn{6}{|c|}{ Knochenmark } \\
\hline 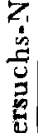 & \multicolumn{2}{|c|}{$\begin{array}{c}\text { Körperg ewich t } \\
\text { (kg) u. } \\
\text { Geschlecht }\end{array}$} & $\begin{array}{l}\mathrm{G}-\mathrm{N} \\
\mathrm{mg} / \mathrm{dl}\end{array}$ & {$\left[\begin{array}{l}\mathrm{R}-\mathrm{N} \\
\mathrm{mg} / \mathrm{d}\end{array}\right.$} & $\mid \begin{array}{c}\text { Eiw. } \\
-\mathrm{N} \\
\mathrm{mg} / \mathrm{d}\end{array}$ & $\left|\begin{array}{c}\text { Alb. } \\
N \\
\mathrm{mg} / \mathrm{d}]\end{array}\right|$ & $\begin{array}{c}\text { Glob } \\
-\mathrm{N} \\
\mathrm{mg} / \mathrm{dl}\end{array}$ & $\left|\begin{array}{c}\text { Fib, } \\
\mathrm{N} \\
\mathrm{mg} / \mathrm{d}\end{array}\right|$ & $\begin{array}{l}\mathrm{G}-\mathrm{N} \\
\mathrm{mg} / \mathrm{dl}\end{array}$ & $\left|\begin{array}{l}\mathrm{R}-\mathrm{N} \\
\mathrm{ng} / \mathrm{d}]\end{array}\right|$ & $\begin{array}{l}\text { Eiw.- } \\
\mathrm{N} \\
\mathrm{mg} / \mathrm{dl}\end{array}$ & $\mid \begin{array}{c}\text { Alb.- } \\
N \\
\mathrm{mg} / \mathrm{dl}\end{array}$ & $\begin{array}{c}\text { Glob } \\
-N \\
\mathrm{mg} / \mathrm{dl}\end{array}$ & $\begin{array}{l}\text { Fib. } \\
N \\
\mathrm{mg} / \mathrm{dl}\end{array}$ \\
\hline 1 & 1,75 & 8 & 988,4 & 203.1 & 785,3 & 3220 & 318,6 & 447 & 10780 & $38=5$ & 205 & 86 & 736 & 6 \\
\hline 2 & 1,80 & 6 & 1156,4 & 327,2 & 829,2 & 384,5 & $|357,6|$ & 87,0 & 1015,0 & 264.5 & 750,5 & 204,0 & 360,0 & 186.5 \\
\hline 3 & 1,55 & ㅇ & 1040,2 & $291,6)^{\prime}$ & 748,5 & 380,2 & 217,9 & 105,0 & 1400,0 & 343,0 & 1057,0 & 345,0 & 358,5 & 353,5 \\
\hline 4 & 1,88 & है & 1047,2 & $|308,1|$ & $|739,1|$ & $|321,2|$ & $|262,1|$ & 155,8 & 931,0 & 260,4 & 670,6 & 199,6 & 225,8 & 245,2 \\
\hline 5 & 1,58 & 우 & 1024,8 & 216,1 & 808,7 & 381,0 & $362,5 \mid$ & 101,2 & 1274,0 & 282,1 & 991,9 & 243,1 & 460,0 & 288,8 \\
\hline 6 & 1,75 & $\hat{\delta}$ & 1089,2 & $319,4 \mid$ & 769,8 & 377,6 & 326,4 & 65,8 & 931,0 & 329,9 & 601,1 & 156,2 & 173,6 & 271,3 \\
\hline & mutm & $\cdots$ & 988,4 & 203,1 & 739,1 & $|321,2|$ & $217,9\}$ & 65,8 & 931,0 & 260,4 & 601,1 & 199,6 & $\mid 173,6$ & 179,6 \\
\hline & ximum & $\ldots$ & 1156,4 & 327,2 & 829,2 & 384,5 & 362,5 & 155,8 & 1400,0 & 338,5 & 1057,0 & 345,0 & 360,0 & 353,5 \\
\hline & rchischnit & $\cdots$ & 1057,7 & $(277,6)$ & 780,1 & 361,1 & 305,1 & 117,5 & 1104,8 & 303,1 & 801,7 & 239,0 & 308,6 & 254,1 \\
\hline & $\begin{array}{l}\text { rchschni } \\
\text { Norm }\end{array}$ & .. & 0,0 & 40,5 & 899,6 & 503,5 & 338,0 & 59,7 & 1605,0 & 119,6 & 1485,4 & 675,0 & 467,2 & 343,2 \\
\hline & $\begin{array}{l}\text { ntisc } \\
\text { hme }\end{array}$ & uüber & & + & $-13,3$ & -28 & - & ${ }^{+} 49$ & $-31,2$ & $+153,4$ & -46 & -64, & $-33, c$ & $-26,0$ \\
\hline
\end{tabular}

Bei Nierenschädigungen nimmt bekanntlich der Rest-(R-) $N$ des Plasmas erheblich zu. Diese R-N-Zunahme wird selbstverständlich durch eine Anomalie des Eiweisstoffwechsels im Gewebe herbeigeführt. An hiesiger Klinik haben Sanada, ${ }^{3)}$ Moriguti11) u.a. an Leber, Niere, Gehirn usw. Zunahme des $\mathrm{R}-\mathrm{N}$ bei Niereninsuffizienz nachgewiesen, aber es war noch nicht geklärt, wie sich der R-N des Knochenmarks dabei verhält.

Nach den Resultaten vorliegender Bestimmungen betrug der R-N, wenn er im Plasma erheblich und zwar bis auf durchschnittlich $277,6 \mathrm{mg} / \mathrm{dl}$ zunahm, im Knochenmark ebenfalls durchschnittlich $303,1 \mathrm{mg} / \mathrm{dl}$, ungefähr $3 \mathrm{mal}$ so viel wie der Normalwert. Aus diesem Befunde ging einleuchtend hervor, dass die Zunahme des Plasma-R-N bei urämischen Zuständen zum Teil auf Zunahme des R-N im Knochenmark, folglich auf Ubertritt des- 
selben in die Blutbahn zurückzuführen ist. Wenn auch der Gesamt-(G-) $\mathrm{N}$ im Plasma ein wenig zunahm, verringerte sich der Eiw.-N infolge erheblicher Zunahme des R-N um 13,3\%. Im Knochenmark zeigte der Eiw.-N eine Abnahme von 46,0\%, da der G-N abnahm und ausserdem der R-N noch zunahm. Was die Fraktionen des Eiw.-N im Plasma anbelangt, betrug der Albumin-(Alb.-) N im Durchschnitt $361,1 \mathrm{mg} / \mathrm{dl}$, der Globulin(Glob.-) $\mathrm{N} 305,1 \mathrm{mg} / \mathrm{dl}$ und der Fibrinogen-(Fib.-) $N$ 117,5 mg/dl. Wenn man im Vergleich mit den normalen Durchschnittswerten die prozentuale $\mathrm{Ab}$ - oder Zunahme berechnet, zeigte der Alb.-N-28,3, der Glob.-N-9,7 und der Fib.- $N+49,1$. Es wurden nämlich absolute und relative deutliche Zunahme des Fib.-N, relative Zunahme des Glob.-N und absolute und relative Abnahme des Alb.-N festgestellt.

Im Knochenmark aber betrug, da der Eiw.-N erheblich abnahm, der Alb.-N durchschnittlich 239,0 mg/dl, der Glob.-N 208,6 mg/dl und der Fib.-N 254,1 mg/dl. Wenn man im Vergleich mit den normalen Durchschnittswerten die prozentuale Abnahme berechnet, so war die Abanhme des Alb.-N am grössten $(-64,6)$. Dann folgte der Glob.-N mit $-33,9$ und der Fib.-N mit -26,0. Im Knochenmark nahmen nämlich sämtliche Fraktionen ab.

Um die Fraktionen des Plasmas und des Knochenmarks vergleichend zu untersuchen und die Beziehungen zwischen ihnen zu prüfen, wurden die prozentualen Verhältnisse der Fraktionen im Eiw.- $\mathrm{N}$ ausgerechnet und

Fig. 1. Prozentuale Verteilung des Alb.Glob.- u. Fib.-N in Eiweiss- $N$ bei Ureterenunterbindung.
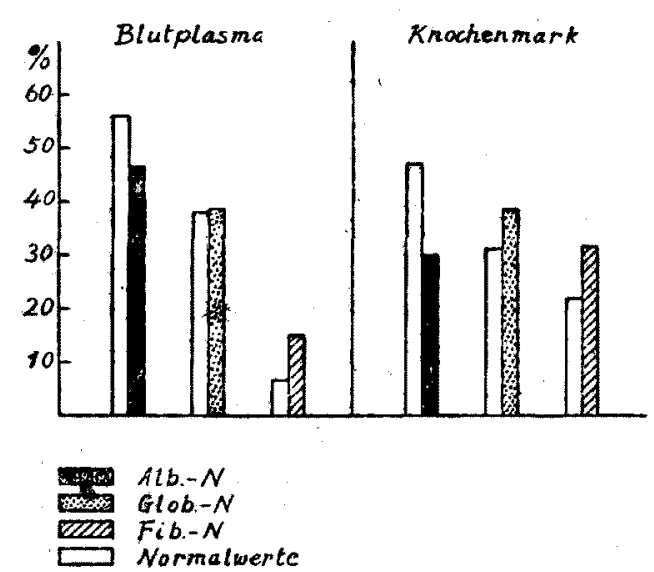
in Fig. 1 wiedergegeten. Wie aus der Figur ersichtlich ist, nahm der Alb.-N sowohl im Plasma als auch im Knochenmark relativ, insbesondere im letzteren erheblich $a b$, der Glob.-N relativ zu, und zwar im Plasma sehr wenig und im Knochenmark auffallend, und der Fib.-N im Plasma wie im Knochenmark deutlich relativ zu.

Die oben erwähnten Veränderungen der Eiweissfraktionen im Plasma, d.h. relative Albuminabund Globulinzunahme, folglich Herabsetzung des. Albumin-Globulin-Quotienten, wurden bereits von einer Reihe Autoren, wie Epstein, ${ }^{12)}$ Ebel, ${ }^{13)}$ Petchacher u. Hönling e $^{14)}$ u.a., und in den Arbeiten hiesiger Klinik von Shida, Oisi $^{(5)} u$. a. nachgewiesen, aber im Knochenmark tritt, wie. Fig. 1 zeigt, diese Neigung noch deutlicher auf. Es wurde von Kollert u. Starlinger, ${ }^{16}$ ) 
$\mathrm{Ozawa}{ }^{17)}$ u.a. nachgewiesen, dass das Plasmafibrinogen bei Niereninsuffizienz zunimmt. Durch vorliegender Bestimmung wurde nun festgestellt, dass es im Knochenmark ebenfalls relativ zunimmt.

Aus dem Gesagten geht hervor, dass bei Niereninsuffizienz auch im Knochenmark ähnliche, und zwar noch deutlichere Veränderungen des Eiweissbildes wie im Blutplasma beobachtet werden können; aus dieser Tatsache wird geschlossen, dass bei Niereninsuffizienz die Ursache für die Veränderungen des R-N sowie der Fraktionen im Blute zum Teil in denselben im Knochenmark zu erblicken ist. Mit anderen Worten, sie deutet darauf hin, dass das Bluteiweiss und das Knochenmarkeiweiss in engster Beziehung zueinander stehen; man muss nämlich das Vorhandensein einer myelo-renalen Korrelation annehmen.

\section{Bestimmungen bei Leberschädigung.}

Da die Herbeiführung von Leberschädigungen durch giftige Substanzen wie Phosphor, Chloroform, Carbontetrachlorid usw. zugleich auch das Knochenmark und andere Organe schädigt, wurden in vorliegender Untersuchung die Choledochusunterbindung und Ligatur der Leberarterien ausgeführt, um möglichst die Leber allein zu schädigen.

\section{(i) Gholedochusunterbindung.}

Gesunden Kaninchen wurde der Ductus choledochus unterbunden, nach ungefähr 72 Stunden, wenn die Symptome des Ikterus manifest auftraten, wurden die Versuchstiere getötet und die Bestimmungen durchgeführt. Die Resultate an 6 Versuchen sind in Tab. II angegeben.

Im Plasma betrug der G-N im Durchschnitt $880,6 \mathrm{mg} / \mathrm{dl}$, nahm also im Vergleich mit den Kontrollversuchen um 6,3\% ab. Der R-N betrug im Durchschnitt $68,6 \mathrm{mg} / \mathrm{dl}$, nahm also gegenüber der Norm um $69,3 \% \mathrm{zu}$. Der Eiw.-N erfuhr durchschnittlich mit $795.4 \mathrm{mg} / \mathrm{dl}$, eine Abnahme von $11,6 \%$, der Alb.-N durchschnittlich mit $396,9 \mathrm{mg} / \mathrm{dl}$, eine solche von $21,2 \%$. Der Glob.-N betrug im Durchschnitt $311,6 \mathrm{mg} / \mathrm{dl}$, nahm also um 7,8\% ab; der Fib.-N durchschnittlich $86,7 \mathrm{mg} / \mathrm{dl}$, nahm also um $45,2 \%$ zu. Im Knochenmark betrug der G-N im Durchschnitt $1276,8 \mathrm{mg} / \mathrm{dl}$, nahm also gegenüber den Kontrollversuchen um 20,5\% ab. Der R-N betrug im Durchschnitt 165,2 mg/dl, nahm also um 38,2\% zu. Der Eiw.-N wies durchschnittlich mit $1111,6 \mathrm{mg} / \mathrm{dl}$, eine Abnahme von 25,2\% auf. Der Alb.- $\mathrm{N}$ betrug durchschnittlich 433,2 mg/dl, mit der Abnahme von 35,8\%. Der Glob.-N betrug durchschnittlich $366,2 \mathrm{mg} / \mathrm{dl}$, nahm also um $21,6 \%$ ab. Der Fib.- $\mathrm{N}$ belief sich durchschnittlich $231,2 \mathrm{mg} / \mathrm{dl}$, einer Abnahme von $9,0 \%$ entsprechend. 
T A B. II.

Eiweissverteilung im Blutplasma u. Knochenmark bei Choledochusunterbindung.

\begin{tabular}{|c|c|c|c|c|c|c|c|c|c|c|c|c|c|c|}
\hline \multicolumn{8}{|c|}{ Blutplasma } & \multicolumn{6}{|c|}{ Knochenmark } & \multirow{2}{*}{ 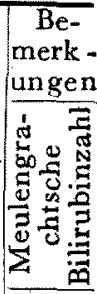 } \\
\hline $\begin{array}{l}\frac{z}{2} \\
\frac{6}{0} \\
\frac{5}{2} \\
\frac{2}{2} \\
2\end{array}$ & $\begin{array}{c}\text { Körper- } \\
\text { gewicht (kg) } \\
\text { u. } \\
\text { Geschlecht }\end{array}$ & $\begin{array}{l}\mathrm{G}-\mathrm{N} \\
\mathrm{mg} / \mathrm{dl}\end{array}$ & $\mid \begin{array}{l}\mathrm{R}-\mathrm{N} \\
\mathrm{mg} / \mathrm{dl}\end{array}$ & $\begin{array}{c}\text { Eiw.- } \\
\mathrm{N} \\
\mathrm{mg} / \mathrm{dl}\end{array}$ & $\mid \begin{array}{c}\mathrm{Alb} .- \\
\mathrm{N} \\
\mathrm{mg} / \mathrm{dl}\end{array}$ & $\mid \begin{array}{c}\text { Glob. } \\
-\mathrm{N} \\
\mathrm{mg} / \mathrm{dl}\end{array}$ & $\left|\begin{array}{c}\text { Fib.- } \\
\mathrm{N} \\
\mathrm{mg} / \mathrm{dl}\end{array}\right|$ & $\begin{array}{c}\mathrm{G}-\mathrm{N} \\
\mathrm{mg} / \mathrm{dl}\end{array}$ & $\begin{array}{c}\mathrm{R}-\dot{\mathrm{N}} \\
\mathrm{mg} / \mathrm{dl}\end{array}$ & $\begin{array}{c}\text { Eiw.- } \\
\mathrm{N} \\
\mathrm{mg} / \mathrm{dl}\end{array}$ & $\left|\begin{array}{c}\mathrm{Alb} .- \\
\mathrm{N} \\
\mathrm{mg} / \mathrm{dl}\end{array}\right|$ & $\left|\begin{array}{c}\text { Glob. } \\
-\mathrm{N} \\
\mathrm{mg} / \mathrm{dl}\end{array}\right|$ & $\begin{array}{l}\text { Fib. } \\
\mathrm{N} \\
\mathrm{mg} / \mathrm{dl}\end{array}$ & \\
\hline 1 & $1,30(1,50)$ 우 & 816,0 & 65,9 & 795,6 & 380,2 & 364,6 & 50,9 & 1322,0 & 262,0 & 1060,0 & 386,4 & 373,3 & 300,3 & 65 \\
\hline 2 & $1,20(1,40)$ 우 & 981,4 & 82,5 & 898,9 & 408,8 & 387,1 & 103,0 & 1246,0 & 117,2 & 1128,8 & 477,4 & 368,9 & 282,5 & 70 \\
\hline 3 & $1,50(1,70) \delta$ & 849,8 & 78,1 & 771,7 & 357,6 & 265,6 & 148,5 & 1200,0 & 161,1 & 1038,9 & 375,4 & 323,5 & 340,0 & 45 \\
\hline 4 & $1,20(1,45)$ 오 & 761,6 & 53,8 & 708,8 & 331,6 & 253,5 & 122,7 & 1212,0 & 164,9 & 1047,1 & 382,3 & 358,7 & 306,1 & 25 \\
\hline 5 & $1,20(1,45)$ s & 772,8 & 58,5 & 714,3 & 438,0 & 230,9 & 45,4 & 1365,0 & 147,5 & 1217,5 & 494,6 & 377,8 & 345,1 & 55 \\
\hline 6 & $1,20(1,45)$ 우 & 956,2 & 72,9 & 883,3 & 465,2 & 368,0 & 50,0 & 1316,0 & 138,9 & 1177,1 & 482,7 & 394,9 & 299,5 & 55 \\
\hline & nimum $\ldots$ & 761,6 & 53,8 & 708,8 & 331,6 & 230,9 & 45,4 & 1200,0 & 117,2 & 1038,9 & 375,4 & 323,5 & 282,5 & $\ldots$ \\
\hline & aximum $\cdots \cdots$ & 981,4 & 82,5 & 898,9 & 465,2 & 387,1 & 148,5 & 1322,0 & 262,0 & 1217,5 & 494,6 & 394,9 & 345,1 & $\cdots$ \\
\hline & Icrhschnitt ... & 880,6 & 68,6 & 795,4 & 396,9 & 311,6 & 86,7 & 1276,8 & 165,2 & 1111,6 & 433,2 & 366,2 & 312,2 & $\cdots$ \\
\hline & $\begin{array}{l}\text { rrchschnitt } \\
\text { 1. Norm }\end{array}$ & 940,0 & 40,5 & 899,6 & 503,5 & 338,0 & 59,7 & 1605,0 & 119,6 & 1485,4 & 675,0 & 467,2 & 343,2 & $\cdots$ \\
\hline & $\begin{array}{l}\text { zentische } \\
\text { od. Zunah- } \\
\text { gegenüber } \\
\text { Jorm }\end{array}$ & 3,3 & + & $-11,6$ & $-21,2$ & $-7,8$ & ${ }^{+} 45,2$ & $-20,5$ & + & $-25,2$ & 35,8 & 21,6 & $-9,0$ & 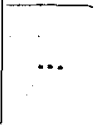 \\
\hline
\end{tabular}

Ziffern in Klammern: Körpergewicht vor d. Choledochusunterbindung.

(ii) Ligatur der Leberarterien.

$\mathrm{Zu}$ diesem Versuche wurden ebenfalls 6 junge gesunde Kaninchen verwendet. Es wurde ihnen die A. hepatica in einer von der Verzweigungsstelle der A. gastroduodenalis direkt distal gelegenen Partie unterbunden, und 48 Stunden danach wurden die Bestimmungen durchgeführt. Die Resultate sind in Tab. III wiedergegeben.

Im Plasma betrug im Durchschnitt der G-N $857,9 \mathrm{mg} / \mathrm{dl}$, der R-N 58,9 mg/dl, der Eiw.-N 809,0 mg/dl, der Alb.-N 389,2 mg/dl, der Glob.-N 315,3 $\mathrm{mg} / \mathrm{dl}$ und der Fib.-N 104,5 mg/dl. Wenn man aus diesen Daten die prozentuale $\mathrm{Ab}$ - oder Zunahme gegenüber den Kontrollwerten berechnet, so zeigt der G-N-7,7, der R-N+45,3, der Eiw.-N-10,1, der Alb.-N-22,7, . der Glob.-N-6,7 und der Fib.-N+74,8.

Im Knochenmark betrug durchschnittlich der G-N 1061,7 mg/dl, der R-N 175,0 mg/dl, der Eiw.-N 886,7 mg/dl, der Alb.-N 329,1 mg/dl, der Glob.-N 291,0 mg/dl und der Fib.-N 266,6 mg/dl. Die prozentuale Aboder Zunahme beläuft sich beim G-N-33,9, beim R-N+46,0 beim Eiw.-N - 40,3, beim Alb.-N-51,2; beim Glob.-N-37,7 und beim Fib.-N-22,0. 
$\mathrm{T}$ A B. III.

Eiweissverteilung im Blutplasma u. Knochenmark bei Leberarterienligatur.

\begin{tabular}{|c|c|c|c|c|c|c|c|c|c|c|c|c|c|c|}
\hline \multicolumn{8}{|c|}{ Blutplasma } & \multicolumn{6}{|c|}{ Knochenmark } & $\begin{array}{c}\text { Be- } \\
\text { merk- } \\
\text { ungen }\end{array}$ \\
\hline$\frac{1}{2}$ & $\begin{array}{c}\text { Körper- } \\
\text { weicht (kg) } \\
\text { u. } \\
\text { Geschlecht }\end{array}$ & $\begin{array}{l}\mathrm{G}-\mathrm{N} \\
\mathrm{ag} / \mathrm{dl}\end{array} \mid$ & $\left|\begin{array}{l}\mathrm{R}-\mathrm{N} \\
\mathrm{mg} / \mathrm{dl}\end{array}\right|$ & $\left|\begin{array}{c}\text { Eiw:- } \\
\mathrm{N} \\
\mathrm{mg} / \mathrm{dl}\end{array}\right|$ & $\left|\begin{array}{c}\text { Alb. } \\
\mathrm{N} \\
\mathrm{mg} / \mathrm{dl}\end{array}\right|$ & $\mid \begin{array}{c}\text { Glob } \\
-\mathrm{N} \\
\mathrm{mg} / \mathrm{dl}\end{array}$ & $\mid \begin{array}{c}\text { Fib.- } \\
\mathrm{N} \\
\mathrm{mg} / \mathrm{dl}\end{array}$ & $\begin{array}{l}\mathrm{G}-\mathrm{N} \\
\mathrm{mg} / \mathrm{dl}\end{array} \mid$ & $\left|\begin{array}{c}\mathrm{R}-\mathrm{N} \\
\mathrm{mg} / \mathrm{dl}\end{array}\right|$ & $\begin{array}{c}\text { Eiw.- } \\
\mathrm{N} \\
\mathrm{mg} / \mathrm{dl}\end{array}$ & $\mid \begin{array}{c}\text { Alb.- } \\
\mathrm{N} \\
\mathrm{mg} / \mathrm{dl}\end{array}$ & $\begin{array}{c}\text { Glob } \\
-\mathrm{N} \\
\mathrm{mg} / \mathrm{dl}\end{array}$ & $\begin{array}{c}\text { Fib.- } \\
\mathrm{N} \\
\mathrm{mg} / \mathrm{dl}\end{array}$ & 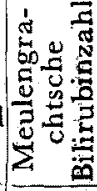 \\
\hline 1 & $1,17(1,45)$ 古 & 960,4 & 66,0 & 894,4 & 399,1 & 366,5 & 128,9 & 966,0 & 169,2 & 796,8 & 328,1 & 210,1 & 258,6 & 7 \\
\hline 2 & $1,05(1,30) \delta$ & 812,0 & 82,5 & 729,5 & 373,2 & 250,0 & 106,3 & 1092,0 & 186,6 & 905,4 & 244,7 & 362,7 & 298,0 & 8 \\
\hline 3 & $1,15(1,40)$ s & 816,2 & 37,3 & 778,8 & 350,7 & 332,8 & 95,4 & 987,0 & 134,5 & 852,5 & 325,5 & 5234,4 & 292,6 & 8 \\
\hline 4 & $1,15(1,45)$ 우 & 984,2 & 43,4 & 940,8 & 489,5 & 346,8 & 104,4 & 1162,0 & 178,0 & 984,0 & 377,4 & 344,9 & 261,7 & 7 \\
\hline 5 & $1,20(1,50) \delta$ & 802,1 & 65,8 & 736,3 & 340,2 & 303,9 & 92,2 & 1122,0 & 199,8 & 922,2 & 372,6 & 301,7 & 247,9 & 8 \\
\hline 6 & $1,10(1,45)$ 우 & 832,4 & 58,5 & 773,9 & 382,6 & 291,6 & 99,6 & 1041,5 & 181,8 & 859,7 & 326,8 & 292,3 & 240,6 & 10 \\
\hline & nimum ... & 802,1 & 73,3 & 729,5 & 340,2 & 250,0 & 92,2 & 966,0 & 134,5 & 796,8 & 244,7 & 210,1 & 240,6 & $\cdots$ \\
\hline & aximum ... & 984,2 & 82,5 & 940,8 & 489,5 & 366,5 & 128,9 & 1162,0 & 199,8 & 984,0 & 372,6 & 362,7 & 298,0 & $\ldots$ \\
\hline & chschnitt & 867,9 & 58,9 & 809,0 & 389,2 & 315,3 & 104,5 & 1061,7 & 175,0 & 886,7 & 329,1 & 291,0 & 266,6 & $\cdots$ \\
\hline & $\begin{array}{l}\text { chschnitt } \\
\text { Norm }\end{array}$ & 940,0 & 40,5 & 899,6 & 503,5 & 338,0 & 59,7 & 1605,0 & 119,6 & 1485,4 & 675,0 & 476,2 & 343,2 & $\ldots$ \\
\hline & $\begin{array}{l}\text { entische } \\
\text { od. Zunah } \\
\text { gegenübe } \\
\text { Torm }\end{array}$ & 7,7 & $+\frac{+}{45,3}$ & $-10,1$ & 22,7 & $-6,7$ & 74,8 & ${ }^{-} 33,9$ & $+_{46,0}$ & $-40,3$ & $-51,2$ & $-37,7$ & $-22,0$ & \\
\hline
\end{tabular}

Ziffern in Klammer: Körpergewicht vor Leberarterienligatur.

Wenn man die Durchschnittswerte und die prozentuale $\mathrm{Ab}$ - oder Zunahme in den Bestimmungsdaten bei den durch die oben erwähnten beiden Methoden herbeigeführten Leberschädigungen betrachtet, so erkennt man eine ganz ähnliche Neigung der Veränderungen, wenn auch ihr Grad in den einzelnen Daten verschieden ist. Der G-N z.B. zeigt, wie bereits von $\mathrm{Ozawa}{ }^{17)} \mathrm{Nakao}$. Matuzaki ${ }^{18)}$ festgestellt worden ist, in vorliegenden Fällen ebenfalls im Plasma geringe Abnahme, im Knochenmark aber erhebliche Abnahme. Der $\mathrm{R}-\mathrm{N}$ nimmt, wie $\mathrm{S}$ ak ai ${ }^{19)} \mathrm{Aoi},{ }^{20)} \mathrm{O}$ is i ${ }^{21}$ u.a. feststellten, im Plasma etwas zu und im Knochenmark ebenfalls ausgesprochen zu. Dass bei Leberschädigungen der R-N, wenn er im Plasma zunimmt, in den Gewebe von Leber, Niere und Hirn ebenfalls zunimmt, ist von $\mathrm{Sanada^{31 }}$ und Moriguti ${ }^{12)}$ nachgewiesen. Da aber hier nachgewiesen worden ist, dass der R-N auch im Knochenmark zunimmt, liegt eine der Ursachen für die Zunahme des R-N im Plasma offenbar in derselben im Knochenmark. Der Eiw.-N zeigt im Plasma geringe Abnahme und im Knochenmark weit stärkere Abnahme. Um die Beziehungen zwischen Alb. -, Glob.- und Fib.-N dabei zu beobachten, wurden die Verhältnisse der Fraktionen im Eiw.- $N^{+}$ausgerechnet und in Fig. 2 (Chole- 
Fig. 2. Prozentuale Verteilung des Alb.-, Glob.- $\mathrm{u}$. Fib.- $\mathrm{N}$ in Eiweiss- $\mathrm{N}$ bei Choledochusunterbindung.

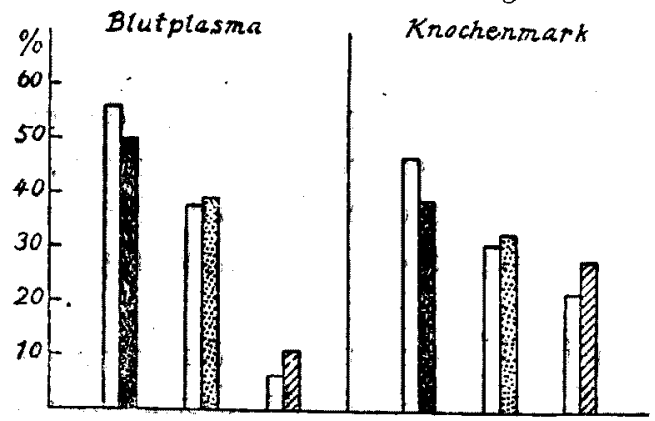

Fig. 3. Prozentuale Verteilung des Alb.-,

Glob.- u. Fib.-N in Eiweiss- $\mathrm{N}$ bei Leberarterienligatur.

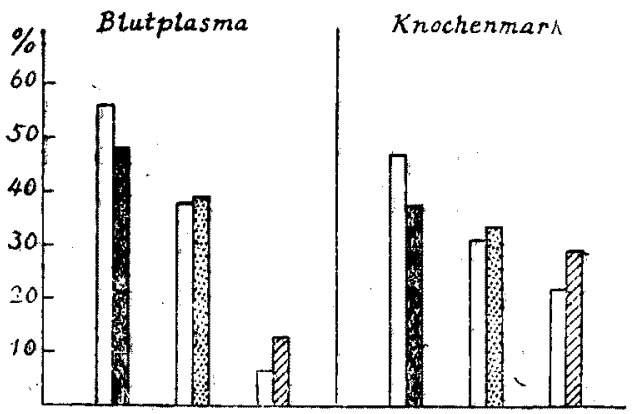

dochusunterbindung) und Fig. 3 (Leberarterienligatur) wiedergegeben Ans den 2 Figuren erkennt man, dass nicht nur die Richtung, sondern auch die Grade der Veränderungen bei Choledochusunterbindung und Leberarterienligatur annähernd gleich sind. Darum wollen wir in der folgenden Darstellung die Resultate der beiedn Schädigungsarten nicht trennen.

Über die Veränderung des Albumin-Globulin-Quotienten im Plasmaeiweiss bei Leberschädigungen sind $\mathrm{zu}$ zahlreiche Berichte erschienen, um sie alle einzeln aufführen zu können. Die Verringerung haben $\mathrm{A} \mathrm{b} \mathrm{e,}{ }^{22}$ ) Yas uda, ${ }^{5)} \mathrm{K}$ u s a n o, ${ }^{23)}$ 'Shida, ${ }^{4)} \mathrm{K}$ os ek i24) $\mathrm{u}$.a. an hiesiger Klinik von der Kolloidosmometrie her nachgewiesen, in vorliegenden Versuchen eine relative Abnahme des Albumins und relative Zunahme des Globulins und Fibrinogens im Plasma bestätigt, wie aus Fig. 2 und 3 ersichtlich ist. Wie die Figuren deutlich zeigen, werden dabei im Knochenmark ganz ähnliche Veränderungen wie im Plasma beobachtet.

Was die Ursache für diẹ Veränderungen des Eiweissbildes im Plasma und Knochenmark anbetrifft, so geht aus dem in die I. Mitteilung ${ }^{10}{ }^{1}$ geschilderten klar hervor, dass das Knochenmark Eiweiss ans Blut liefert, so dass dürfte man nicht annehmen, dass es sich hier um einen ganz passiven Vorgang handeln, wobei die Veränderungen der Eiweissfraktion im Blute sekundär auf dem Eiweissbild des Knochenmarks ausgewirkt haben sollte, die Annahme liegt hier nahe, dass das Knochenmarkeiweiss sich unter Wirkung der durch Leberschädigung erzeugten Substanzen oder der humoralen sowie anderen myelohepatalen Korrelationen primär qualitativ ver. ändert und relative Zunahme des Globulins und Fibrinogens sowie relative Abnahme des Albumins verursacht, und wobei der Globulin urrd Fibrinogen ins Blutplasma übertretend relative Zunahme derselben und die relative Abnahme des Albumins im Plasma bewirken. Auf Grund dieser Tatsache darf man wohl sagen, dass das Knochenmark auch bei Leberschädigung wie bei der der Nieren die Veränderungen des Bluteiweisses bedeutend beeinflusst. Mit anderen Worten, soweit es das Bluteiweiss betrifft, kann man 
wohl das Vorhandensein einer myelo-renalen und einer myelo-hepatalen Korrelation annehmen.

\section{ZUSAMMENFASSUNG.}

An normalen jungen Kaninchen wurden Nierenschädigungen und Leberschädigungen durch Choledochus- oder Leberarterienligatur hervorgerufen und die Veränderungen der Eiweissfraktionen im Knochenmark bestimmt. Beim Vergleich mit den Eiweissfraktion des Plasmas gelangt man zu folgenden Ergebnissen;

1. Bei Nierenschädigungen treten im Knochenmark Abnahme des Gesamt- (G-)N, erhebliche Zunahme des Rest-(R-)N und deutliche Abnahme des Eiw.-N auf. Hinsichtlich der Fraktionen des Eiw.-N sind relative Abnahme des Albumins und relative Zunahme des Globulins und Fibrinogens auftallend. Wenn auch die Richtung dieser Veränderungen im grossen und ganzen derjenigen im Plasma ähnlich ist, ist ihr Grad viel erheblicher als der im Plasma.

2. Bei Leberschädigungen zeigen sich im Knochenmark Abnahme des G-N, Zunahme des R-N und Abnahme des Eiw.-N; diese Tendenzen stimmen mit den Veränderungen im Plasma überein. Was die Fraktionen des Eiw.-N anbelangt, nimmt das Albumin relativ ab; Globulin und Fibrinogen nehmen dagegen relativ zu. Die Richtung und das Ausmass dieser Veränderungen sind annähernd gleich denen im Plasma.

3. Aus dem Gesagten geht hervor, dass die Ursache für die Veränderungen der einzelnen Eiweisskörper im Plasma bei Nieren- oder Leberschädigung zum Teil im Knochenmark zu erblicken ist. Man kann nämlich annehmen, dass bezüglich des Verhaltens der Eiweisskörper sich myelo-renale und eine myelo-hepatale Korrelation nachweisen lässt.

\section{Literatur.}

1. Häbler, Günther, Scheiner, Wagner u. Werner, Ztschr. f. ges. exp. Med., 1934, 94, 596.

2. K. K i mura u. N a ka z aw a, Tohoku Journ. Exp. Med., 1930, 16, 352.

3. S a n a d a, Ibid., 1936, 29, 156, 202.

4. Shida, Ibid., 1939, 35, 304, 445 .

5. Yasuda, Ibid., 1937, 31, 456, 524 .

6. Nolf, Arch. internat. de Physiol., 1905, 3, 1.

7. Whi p pleu. Hurwitz, Journ. Exp. Med., 1911, 13, 136.

8. Ta n a ka, Fukuoka Ikadaigaku Zassi, 1928, 21, 948.

9. Mann u.. Magath, Arch. intern. Med., 1922, 30, 73.

10. Ni hei, Tohoku Journ. Exp. Med, 1947, 49, 39.

11. Moriguti, Ibid., 1942, 42, 358.

12. Epstein, Jorun. Exp. Med., 1912, 15, 485. 
13. E bel, Monatsschr. f, Kinderheilk., 1925, 29, 116.

14. Petchacher u. Hönlinger, Wien. Arch. f. inn. Med., 1925, 9, 357.

15. O isi, Tohoku Journ. Exp. Med., 1941, 39, 435.

16. Kollertu. St arlinger, Wien. klin. Wschr., 1922, 35, 439.

17. Oz a wa, Fukuoka Ikadaigaku Zassi, 1926, 19, 701.

18. Nakao, Kin u. Mat u z aki, Chosen Igakuzassi, 1933, 23, i758.

19. S a ka a, Zikken Syokakibyogakkai Zassi, 1930, 5, 640 .

20. A o i, Aiti Igakkai Zassi, 1930, 37, 2826.

21. O i si, Tohoku Journ. Exp. Med., 1941; 39, 462.

22. A b e, Ibid., 1931, 17, 412 .

23. Kus a no, Ibid., 1938, 34, 57.

24. Kos e ki, Ibid., 1941, 39, 580 . 